\title{
Self-Loosening Failure Analysis of Bolt Joints under Vibration considering the Tightening Process
}

\author{
Yan Chen, Qiang Gao, and Zhenqun Guan \\ State Key Laboratory of Structural Analysis for Industrial Equipment, Department of Engineering Mechanics, Dalian University of \\ Technology, Dalian 116024, China
}

Correspondence should be addressed to Zhenqun Guan; guanzhq@dlut.edu.cn

Received 19 July 2017; Revised 20 October 2017; Accepted 24 October 2017; Published 6 December 2017

Academic Editor: Sergio De Rosa

Copyright (C) 2017 Yan Chen et al. This is an open access article distributed under the Creative Commons Attribution License, which permits unrestricted use, distribution, and reproduction in any medium, provided the original work is properly cited.

By considering the tightening process, a three-dimensional elastic finite element analysis is conducted to explore the mechanism of bolt self-loosening under transverse cyclic loading. According to the geometrical features of the thread, a hexahedral meshing is implemented by modifying the node coordinates based on cylinder meshes and an ABAQUS plug-in is made for parametric modeling. The accuracy of the finite element model is verified and validated by comparison with the analytical and experimental results on torque-tension relationship. And, then, the fastening states acquired by different means are compared. The results show that the tightening process cannot be replaced by a simplified method because its fastening state is different from the real process. With combining the tightening and self-loosening processes, this paper utilizes the relative rotation angles and velocities to investigate the slip states on contact surfaces instead of the Coulomb friction coefficient method, which is used in most previous researches. By contrast, this method can describe the slip states in greater detail. In addition, the simulation result reveals that there exists a creep slip phenomenon at contact surface, which causes the bolt self-loosening to occur even when some contact facets are stuck.

\section{Introduction}

The bolt joint, as a very common component in engineering, is widely used in a variety of industrial machines because of its simple configuration, convenient operation, and low cost [1]. A typical diagram of the bolt joint structure is shown in Figure 1. In practical applications, the bolt joint always requires a sufficiently large preload to guarantee a reliable force transmission between the clamped components. However, due to the complex working environment, bolt joints often experience self-loosening (gradual loss of preload) with increasing service time, which can cause a decrease in the structure stiffness and in some cases may even lead to fatal consequences if it remains undetected [2].

In 1969, Junker [3] first indicated that transverse or shear loading (perpendicular to the fastener axis) is the most dangerous form of loading on self-loosening. Since then, self-loosening of bolted joints under cyclic transverse loads has become a popular topic in the study of bolt science. Pai and Hess $[4,5]$ introduced the concept of localized slip and classified the self-loosening process into four different types: (1) localized head slip with localized thread slip; (2) localized head slip with complete thread slip; (3) complete head slip with localized thread slip; (4) complete head slip with complete thread slip. However, the friction coefficients on the two surfaces are usually close, and, in this case, the complete thread slip is achieved prior to bolt head slip, which leads to the rare occurrence of the third type $[6,7]$. Kasei $[8]$ and Izumi et al. [9] investigated the mechanism of self-loosening due to micro bearing-surface slip. The results showed that a small degree of loosening occurs when transverse load reaches the range 50 to $60 \%$ of the critical loading for the bearing-surface slip. Recently, the cycle rotation load has been accepted as another reason to cause self-loosening [10, 11]. The results by Yokoyama et al. [10] revealed that loosening occurs only when the rotation angle around bolt axis which is applied to clamped component reaches a critical value, and the thread surface undergoes a complete slip. For a further understanding of localized slip and complete slip at contact surfaces, Dinger and Friedrich [7] proposed a local 


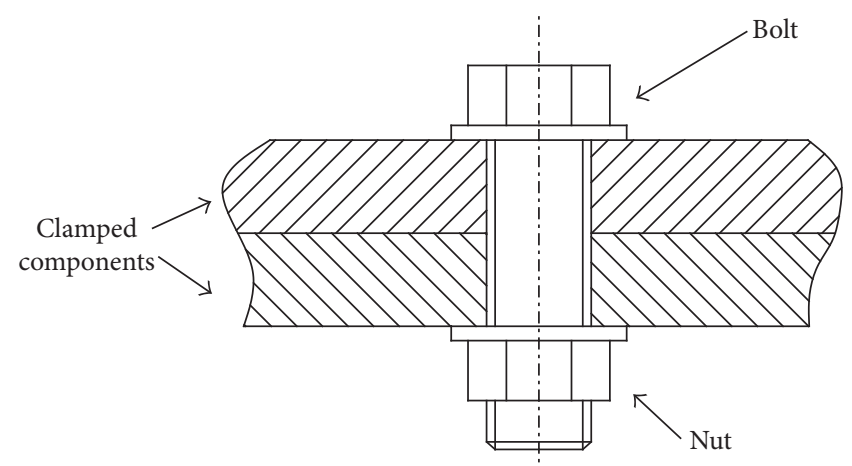

Figure 1: A typical bolt joint diagram.

key parameter $\eta_{n}$ (obtained by (1)) to characterize the contact situation; $\eta_{n}=0 \%$ represents no contact; $0 \%<\eta_{n}<100 \%$ represents sticking at the contact node; $\eta_{n}=100 \%$ represents slipping at the contact node. In (1), $\tau$ is the shear stress; $p$ is the contact stress; and $\mu$ is the friction coefficient.

$$
\eta_{n}=\frac{\tau}{p \cdot \mu} \times 100 \%
$$

Through the same method, Jiang et al. [12] focused on the self-loosening of bolts in curvic coupling and noted that the slip states at contact surfaces highly depend on the values of the preload of the bolt and the torque loads on the disc. Additionally, Nassar and Housari [13, 14] used a simplified mathematical model to investigate some factors on self-loosening of threaded fasteners such as the hole clearance, thread fit, thread pitch, and initial tension. In subsequent work, Nassar et al. [15-17] proposed a more accurate mathematical model to predict the variation of the preload during the self-loosening process by investigating the relationship between the bearing friction torque, the thread friction torque, and the pitch torque components. However, simplifications were used in the model that led to its difference from the real structure.

With the development of computer technology, the finite element method is accepted as the most useful numerical method for solving the bolt self-loosening problem [18]. However, it is difficult to model and mesh the helical structure considering the effects of the lead angle. There are two main approaches: one is to model the bolt body and the thread separately and then connect them using a tie constraint [19, 20]. In this way, the two parts can be modeled with hexahedral meshes. However the transmission of force and displacement on the interface is achieved by the interpolation, which results in low accuracy, critical discontinuous stress, and even incorrect high stress at some nodes. The other approach treats the bolt body and the threads as a whole part, which can be meshed only by tetrahedron $[9,10,21,22]$. Unfortunately, a tetrahedron is less accurate and more time-consuming in calculation, compared with the hexahedral mesh. Fukuoka generalized the mathematical expressions of helical thread through the analysis of geometry and proposed a feasible hexahedral mesh generation method [23, 24]; nevertheless, it is complex and cumbersome.
In this paper, we implement the hexahedral mesh generation of the thread structure by modifying the node coordinates. Besides, an ABAQUS plug-in is made for parametric modeling and further study. Using this model, we study the differences between different fastening means and their effects on bolt self-loosening. Additionally, the mechanism of bolt self-loosening is analyzed using the relative motion of nodes and a creep slip phenomenon is illustrated.

\section{Finite Element Model}

2.1. Mathematical Expressions of the Thread Cross-Section Profile. According to the geometry features of the thread, shown in Figure 2, the shape is naturally identical at any crosssection along the bolt axis. To another cross-section, it can be obtained just by rotating a certain angle around the axis based on Figure 2. The surface of the external thread consists of four parts: $A-B$ (thread shank), $B-C$ (crest), $C-D$ (thread shank), and $D-A$ (root radius). The outer contour line of the crosssection is equivalent to the whole pitch. Figure 3 shows the cross-section profile along the bolt axis, including the thread root radius.

Assume that the diagram given by Figure 2 is the crosssection at $z=0$, which can be viewed as a datum plane. The distance between the outer contour line and the axis of the bolt can then be expressed by the following equations:

$$
\begin{aligned}
& r(\theta, z=0) \\
& = \begin{cases}\frac{d}{2}-\frac{7}{8} H+2 \rho-\sqrt{\rho^{2}-\frac{P^{2}}{4 \pi^{2}} \theta^{2},} & \left(0 \leq \theta \leq \theta_{1}\right), \\
\frac{H}{\pi} \theta+\frac{d}{2}-\frac{7}{8} H, & \left(\theta_{1} \leq \theta \leq \theta_{2}\right), \\
\frac{d}{2}, & \left(\theta_{2} \leq \theta \leq \theta_{3}\right), \\
\frac{H}{\pi}(2 \pi-\theta)+\frac{d}{2}-\frac{7}{8} H, & \left(\theta_{3} \leq \theta \leq \theta_{4}\right), \\
\frac{d}{2}-\frac{7}{8} H+2 \rho-\sqrt{\rho^{2}-\frac{P^{2}}{4 \pi^{2}}(2 \pi-\theta)^{2}}, & \left(\theta_{4} \leq \theta \leq 2 \pi\right),\end{cases} \\
& \theta_{1}=\frac{\sqrt{3} \pi \rho}{P}, \\
& \theta_{2}=\frac{7 \pi}{8} \text {, } \\
& \theta_{3}=\frac{9 \pi}{8} \text {, } \\
& \theta_{4}=2 \pi-\theta_{1} \text {, }
\end{aligned}
$$




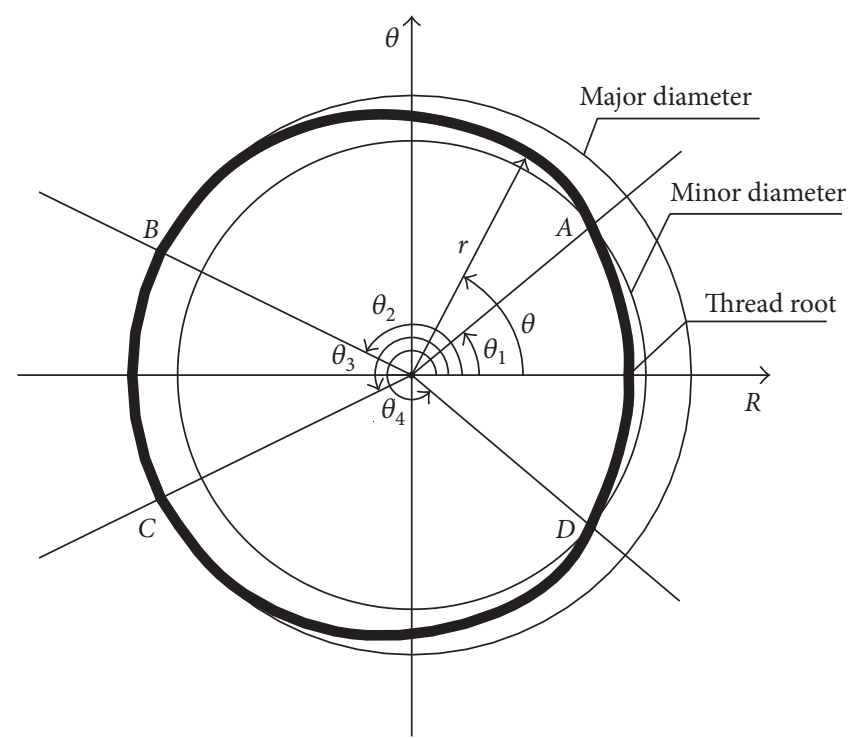

FIGURE 2: The cross-section profile of external thread.

$$
\begin{aligned}
& \rho=\frac{\sqrt{3} P}{12}, \\
& H=\frac{\sqrt{3} P}{2},
\end{aligned}
$$

where $P$ and $d$ represent the thread pitch and the nominal diameter, respectively. $\rho$ is the root radius of the thread. Another cross-section, which is a distance $z$ off the datum plane, has the same shape as the datum one. However, in a cylindrical coordinate, it must be rotated by an angle $\varphi$, which can be written as

$$
\varphi=\frac{2 \pi}{P} z
$$

In addition, it can be known that the mathematical expression of the outer surface of thread is periodic.

$$
r(\theta, z)=r(\theta+2 n \pi, z+m P) .
$$

In summary, the complete expression of the thread crosssection profile can be expressed as follows:

$$
\begin{aligned}
& r(\theta, z) \\
& = \begin{cases}\phi=\theta+\varphi=\theta+\frac{2 \pi}{P} z, & \left(0 \leq \phi \leq \theta_{1}\right), \\
\frac{d}{2}-\frac{7}{8} H+2 \rho-\sqrt{\rho^{2}-\frac{P^{2}}{4 \pi^{2}} \theta^{2},} & \left(\theta_{1} \leq \phi \leq \theta_{2}\right), \\
\frac{H}{\pi} \theta+\frac{d}{2}-\frac{7}{8} H, & \left(\theta_{2} \leq \phi \leq \theta_{3}\right), \\
\frac{d}{2}, & \left(\theta_{3} \leq \phi \leq \theta_{4}\right), \\
\frac{H}{\pi}(2 \pi-\theta)+\frac{d}{2}-\frac{7}{8} H, & \left(\theta_{4} \leq \phi \leq 2 \pi\right), \\
\frac{d}{2}-\frac{7}{8} H+2 \rho-\sqrt{\rho^{2}-\frac{P^{2}}{4 \pi^{2}}(2 \pi-\theta)^{2}},\end{cases}
\end{aligned}
$$

$\theta_{1}=\frac{\sqrt{3} \pi \rho}{P}$,

$\theta_{2}=\frac{7 \pi}{8}$,

$\theta_{3}=\frac{9 \pi}{8}$,

$\theta_{4}=2 \pi-\theta_{1}$,

$\rho \leq+\frac{\sqrt{3} P}{12}$.

Similarly, the profile of the internal thread can be expressed in the same manner and it also has the periodical piecewise function form.

$r^{\prime}(\theta, z)=r^{\prime}(\theta+2 n \pi, z+m P)$,

$r^{\prime}(\theta, z)$

$$
\begin{aligned}
& = \begin{cases}\phi=\theta+\varphi=\frac{2 \pi}{P} z, & \\
\frac{d_{1}}{2}=\frac{d}{2}-\frac{5}{8} H, & \left(0 \leq \theta \leq \theta_{1}^{\prime}\right), \\
\frac{H}{\pi} \theta+\frac{d}{2}-\frac{7}{8} H, & \left(\theta_{1}^{\prime} \leq \theta \leq \theta_{2}^{\prime}\right), \\
\frac{d}{2}+\frac{7}{8} H-2 \rho^{\prime}+\sqrt{\rho^{\prime 2}-\frac{P^{2}}{4 \pi^{2}}(\pi-\theta)^{2}}, & \left(\theta_{2}^{\prime} \leq \theta \leq \theta_{3}^{\prime}\right), \\
\frac{H}{\pi}(2 \pi-\theta)+\frac{d}{2}-\frac{7}{8} H, & \left(\theta_{3}^{\prime} \leq \theta \leq \theta_{4}^{\prime}\right), \\
\frac{d_{1}}{2}, & \left(\theta_{4}^{\prime} \leq \theta \leq 2 \pi\right),\end{cases} \\
& \theta_{1}^{\prime}=\frac{\pi}{4}, \\
& \theta_{2}^{\prime}=\pi\left(1-\frac{\sqrt{3} \rho^{\prime}}{P}\right),
\end{aligned}
$$




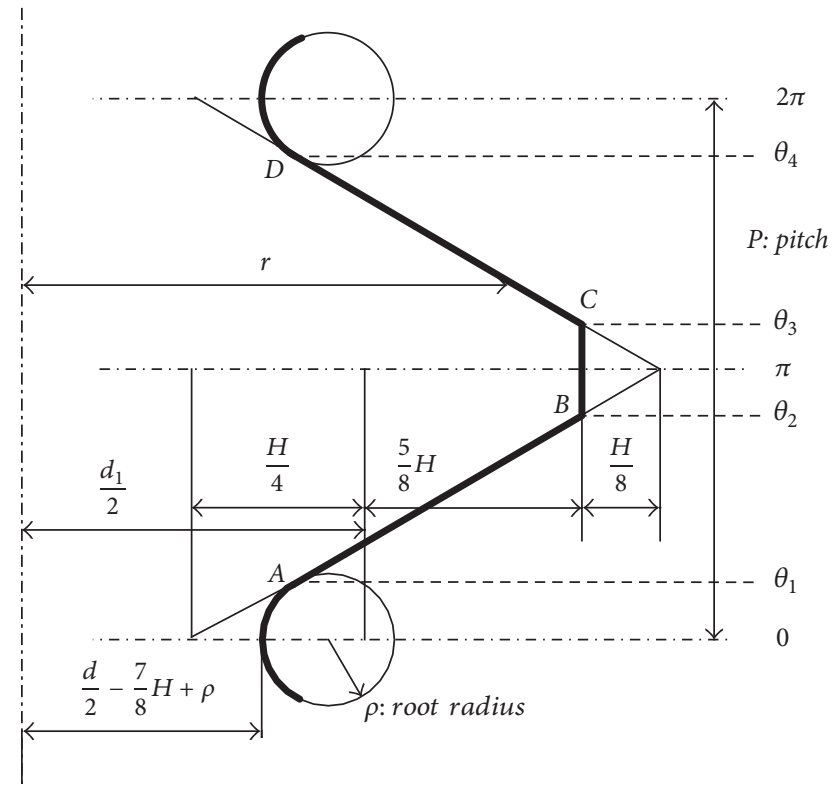

FIgURE 3: The cross-section profile along the bolt axis.

$$
\begin{aligned}
& \theta_{3}^{\prime}=\pi\left(1+\frac{\sqrt{3} \rho^{\prime}}{P}\right), \\
& \theta_{4}^{\prime}=\frac{7 \pi}{4} \\
& \rho^{\prime} \leq \frac{\sqrt{3}}{24} P .
\end{aligned}
$$

2.2. Automatic Mesh Generation of the Thread Structure. The mesh generation method Fukuoka proposed requires many cyclic operations on the two-dimensional elements such as "copy"; "translate"; "rotate"; and "merge," which easily cause the problem of expensive computation. In this study, the thread structure is partitioned properly before meshing. The hexahedral mesh generation is implemented by modifying the node coordinates based on cylindrical hexahedron meshes. The detailed procedures are introduced below. In addition, a self-developed ABAQUS plug-in is made for parametric modeling (Figure 4).

Step 1. Depending on the size of the bolt and nut, the corresponding cylinders are modeled with hexahedral meshes in ABAQUS. To fit the shape of the thread well and improve the calculation efficiency, the model is divided into two parts: the thread region and the nonthread region. The thread region is discretized with finer meshes to guarantee the simulation accuracy of the contact state, while the other region is meshed with relative coarse elements (Figure 5). The models are then exported in the form of an INP file.

Step 2. The node coordinates of the thread region are extracted from the INP file exported previously, and they are modified by a self-compiled program depending on the

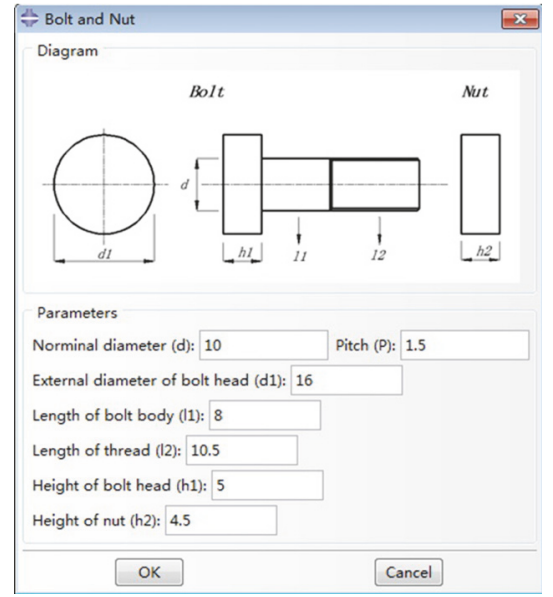

FIgURE 4: The parametric modeling interface.

mathematical expression of thread cross-section profile. The modified INP file is then imported to ABAQUS again, and the hexahedral models of threads are generated (Figure 6).

Step 3. The bolt shank and bolt head, which are simple cylinders, are built up and meshed with hexahedral elements. Then they are merged with the thread part to obtain a complete bolt model.

Step 4. Finally, the clamped components are modeled in ABAQUS, and all parts are assembled into a whole analysis model of the bolt self-loosening problem (Figure 7).

Through the method described before, the 3D finite element model, which contains a $\mathrm{M} 10 * 1.5$ bolt and nut and two clamped components, is established using the commercial software package ABAQUS. There are a total of 58140 C3D8 elements and 64731 nodes. All components in this model are assumed to be purely elastic, and Young's modulus, Poisson's ratio, and the density are $210 \mathrm{GPa}, 0.3$, and $7800 \mathrm{~kg} / \mathrm{m}^{3}$, respectively.

Contact interactions have been set between all sliding surfaces, including the interfaces between the threads, bolt underhead surface and the upper part surface, and nut surface and the lower part surface. Contact modeling is very important to the simulation of the tightening and self-loosening process. According to the work by Dinger and Friedrich [7], the augmented Lagrangian technique and the penalty approach are applied to solve the normal and tangential contact problem, respectively. In this study, the surface constraint approach used for all contact pairs is a master-slave, finite sliding, and node-to-surface assignment. Concretely, to the interface between internal and external threads, the surface of external threads is assigned the master surface; to the interface between bolt and upper component, the surface of upper component is assigned the master surface; to the interface between nut and lower component, the surface of lower component is assigned the master surface; to the interface between clamped components, the surface of lower component is assigned the master surface. 


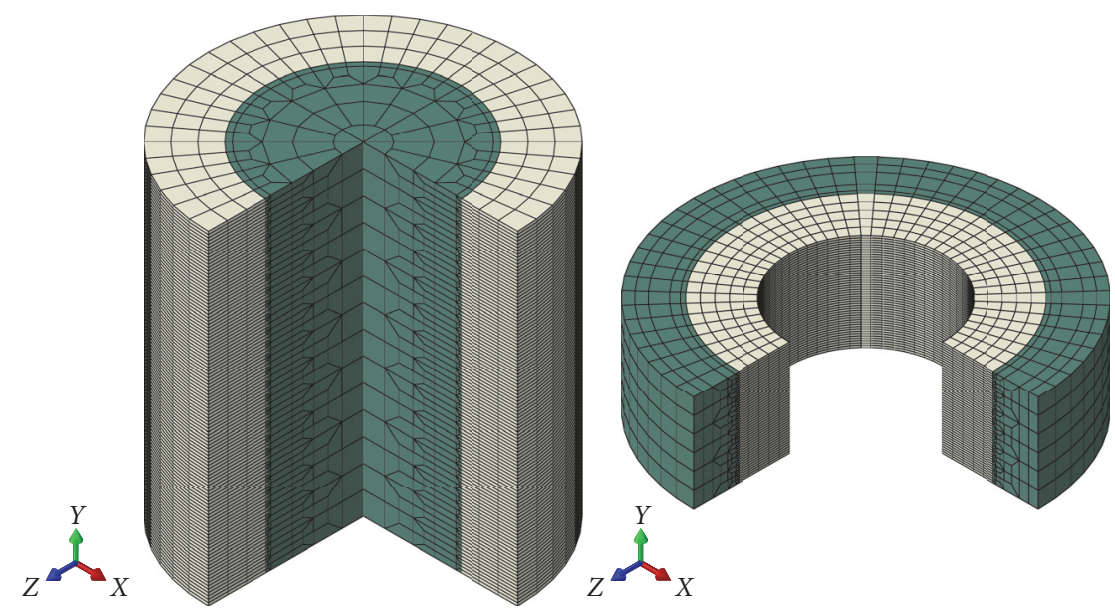

Figure 5: The hexahedral models of cylinders.

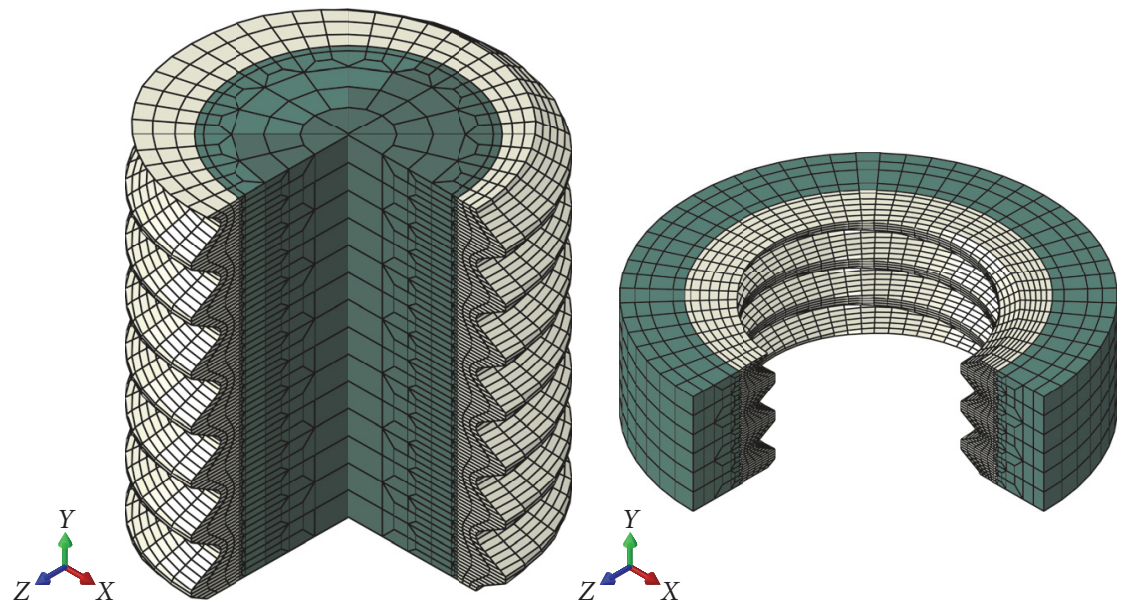

Figure 6: The hexahedral models of threads.
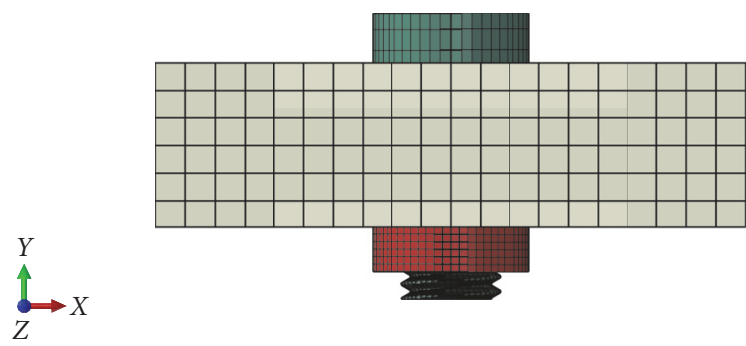

Figure 7: The whole hexahedral model of a typical bolt joint.

A nominal friction coefficient 0.1 is initially used for all sliding surfaces. Considering the geometric nonlinearity, the implicit dynamics module in ABAQUS is used to simulate the tightening and self-loosening process.

\section{Analysis of the Tightening Process}

3.1. Verification of the Accuracy of the FE Model. The preload refers to the elastic restoring force of the bolt when it is serving. In engineering, it is always controlled by tightening torque or rotation angle of the nut. In this paper, the tightening process analysis is performed by applying a ramp circumferential displacement $\theta_{0}$ on the side surface of the nut (Figure 8) and then removing it after maintaining the value for a period. The accuracy of finite element model is verified by contrast with the analytical and experimental results on torque-tension relationship.

Referring to the general rules of tightening for threaded fasteners given in GB/T 16823.2 [25], the relationship between tightening torque $T_{f}$ and bolt preload $F_{f}$ can be estimated approximately by

$$
T_{f}=K F_{f} d,
$$

where $K$ is the torque coefficient and $d$ is the nominal diameter of the bolt. $K$ can be expressed as follows:

$$
K=\frac{1}{2 d}\left(\frac{P}{\pi}+\mu_{s} d_{2} \sec \alpha^{\prime}+\mu_{W} D_{W}\right),
$$

where $P$ is the thread pitch, $\mu_{s}$ is the coefficient of thread friction, $d_{2}$ is the intermediate diameter, $\alpha^{\prime}$ is half of the 


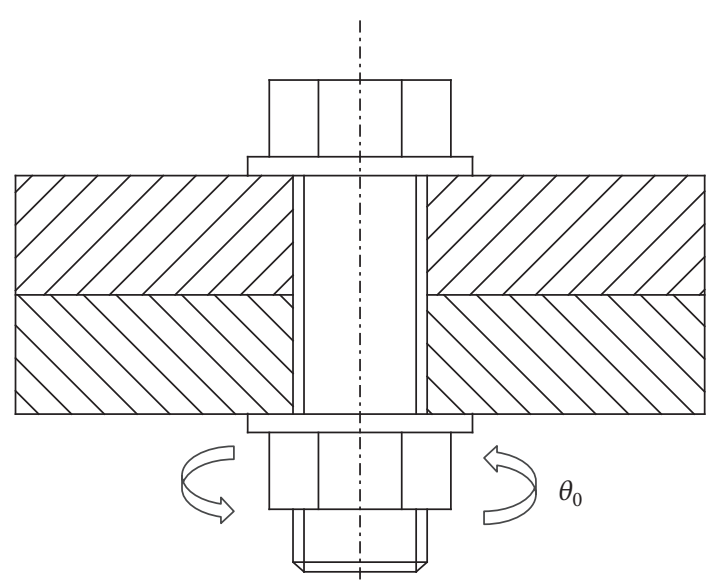

FIgURE 8: The loading diagram.

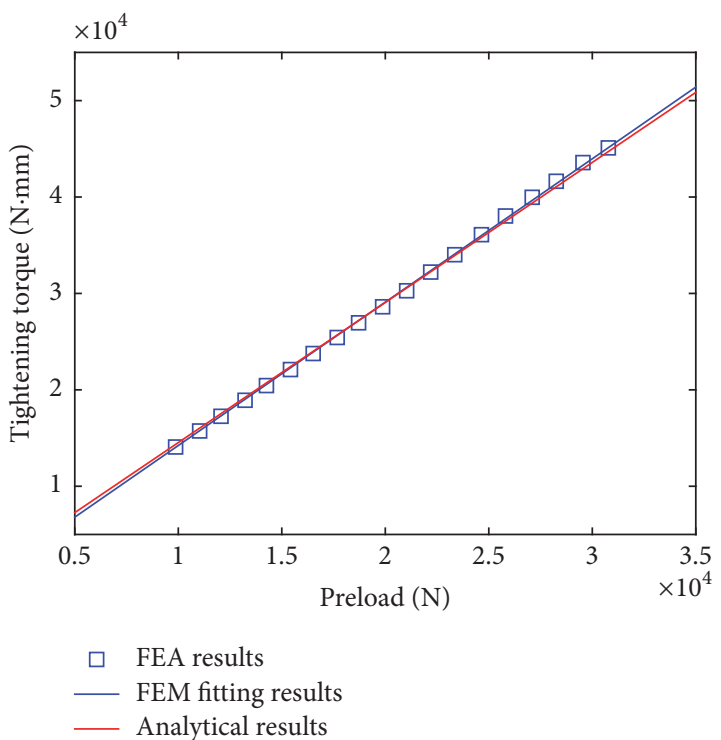

FIGURE 9: The contrast diagram of torque-tension relationships.

thread profile angle (30 degrees for standard ISO threads), $\mu_{W}$ is the coefficient of underhead friction, and $D_{W}$ is the effective underhead bearing contact radius, which can be expressed as follows when the underhead bearing face is circular:

$$
D_{W}=\frac{2}{3} \times \frac{d_{W}^{3}-d_{h}^{3}}{d_{W}^{2}-d_{h}^{2}}
$$

where $d_{h}$ and $d_{W}$ are the internal diameter and the external diameter of the underhead bearing contact face, respectively.

To a $\mathrm{M} 10 * 1.5$ bolt and nut, the torque-tension relationship can be acquired by substituting relevant parameters into (7)-(9). Figure 9 shows the comparison between the analytical results and simulation results. It can be seen that the FEA (finite element analysis) results are similar to the analytical ones. The torque coefficient $K$ by the analytical method is 1.453 , while the value is 1.4749 by FEM (finite element method). The error between them is only $1.51 \%$.
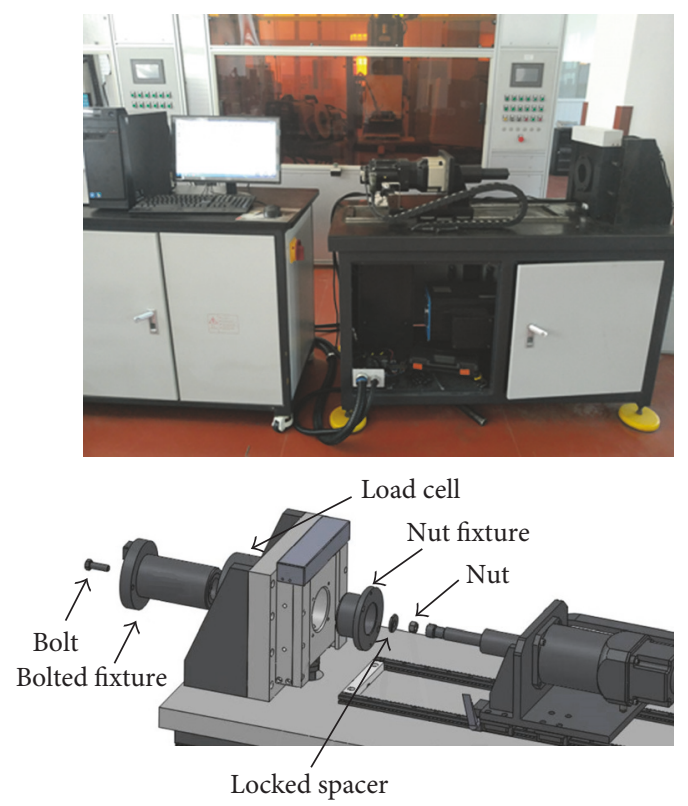

Figure 10: The test rig.

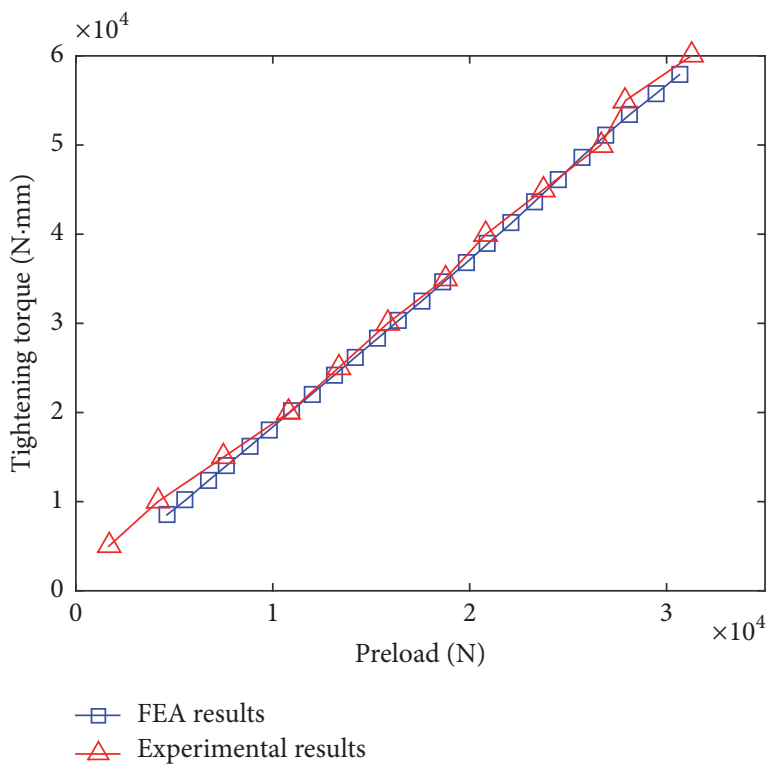

FIGURE 11: Comparison between the finite element method and the experimental method.

In addition, a test rig has been developed as shown in Figure 10 with an aim to verify the accuracy of the FE model. In this experiment two plates made of steel (30CrMnSiA) are clamped by a M10 * 1.5 bolt and nut. Each plate has a thickness of $10 \mathrm{~mm}$. During the loading process, the bolt head is fixed and a torque wrench is used to tighten the nut. The value of tightening torque is applied in the range of $0 \sim 60 \mathrm{Nm}$. Besides, a load cell is attached between the bolt head and the clamped component to measure the clamp force. The data of fastener tension are recorded per $5 \mathrm{Nm}$ torque in this experiment.

The curves in Figure 11 show the contrast results between finite element method and the experimental method. 


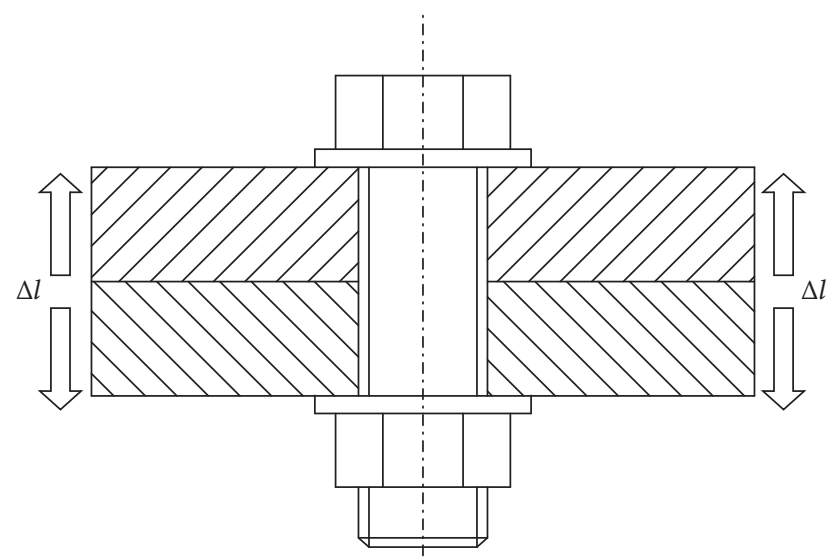

FIGURE 12: The loading diagram of the simplified way.

Through the comparison, it can be seen that the difference between the finite element and the experimental results is tiny. The accuracy of FEM is verified by analytical and experimental study.

3.2. Analysis of the Fastening States. The preload is a very important factor that should not be ignored in the study of bolt self-loosening. However, few studies considered both the tightening process and the self-loosening process simultaneously. Most of them simulated the preload by stretching the bolt or using a cooling pretightening algorithm that makes the fastening state of bolt different from the real case. Therefore, the differences of different fastening ways are discussed here, followed by their effects on bolt selfloosening.

To simulate the tightening process, a circumferential displacement $\theta_{0}$ is applied on the side surface of the nut, which is the same as the model validation process. Besides, a simplified way that stretches the bolt is made for comparison. The two clamped components are separated by a distance $\Delta l$, which is used to control the value of preload, as shown in Figure 12. After the loading process, the whole bolt joint becomes static without external constraints and the resultant torque at every contact surface is equal in magnitude. So the resultant torque at contact surface between threads is regarded as the object. Figure 13 presents the relationships between the torque and the preload in static state, which are achieved by different fastening means.

As shown before, the value of the torque in the tightening process is obviously higher than that obtained by stretching the bolt under the same preload, which leads to making the self-loosening more likely to occur. The resultant torque on the thread interface consists of two parts: the pitch torque and the thread friction torque. Figure 14 illustrates the relationships between the preload, the pitch torque, the thread friction torque, and the resultant torque by different fastening means. It can be noted that the pitch torque and thread friction torque possess the same direction when considering the tightening process, while it is opposite by

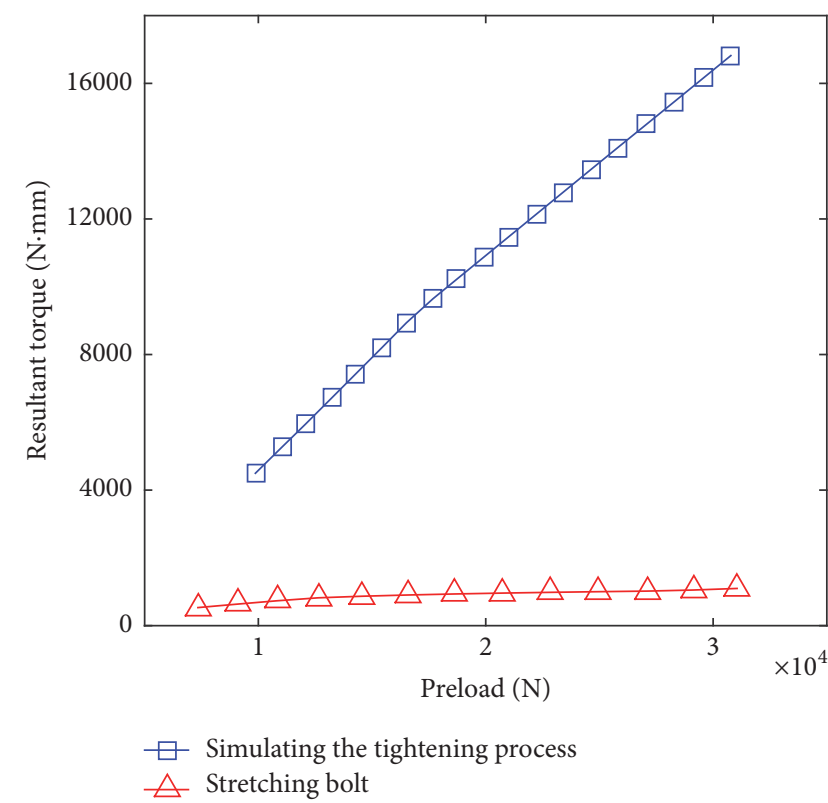

FIGURE 13: The relationships between the torque and the preload.

stretching the bolt, which leads to a much smaller resultant torque.

\section{Analysis of the Bolt Loosening Mechanism}

Considering the influence of the tightening process, the preload is produced by applying a constrained circumferential displacement on the side surface of the nut, followed by its removal. To conduct the FEA of bolt joints self-loosening, a transversal excitation $s$ is loaded on the clamped components (Figure 15), which is determined by the following formula:

$$
s=s_{0} \sin (\omega t)
$$

where $s_{0}$ and $\omega$ represent the excitation amplitude and angular frequency, which are $0.03 \mathrm{~mm}$ and $2 \pi$ in this paper, respectively. The excitation amplitude is smaller than the clearance between the bolt body and the clamped part. To reduce computational cost, the clamped components are assumed to be rigid bodies when simulating the selfloosening process of bolt joints. Since the vibration frequency is set to $1 \mathrm{~Hz}$, which is low, the system can be treated as a quasi-static process. Because the self-loosening behavior is mainly caused by slip at contact surfaces, the critical output parameters monitored in this analysis include the preload and the motion of nodes at contact surfaces, which are used to analyze the slip state.

4.1. Evolution of the Preload during Self-Loosening. To investigate the effects of different fastening means on the selfloosening process of the bolt joint, the same preload is produced by adjusting the circumferential displacement and the separation distance in finite element analysis, and the other attributes of the two models are completely identical. A cyclic transversal displacement is then loaded on the clamped 


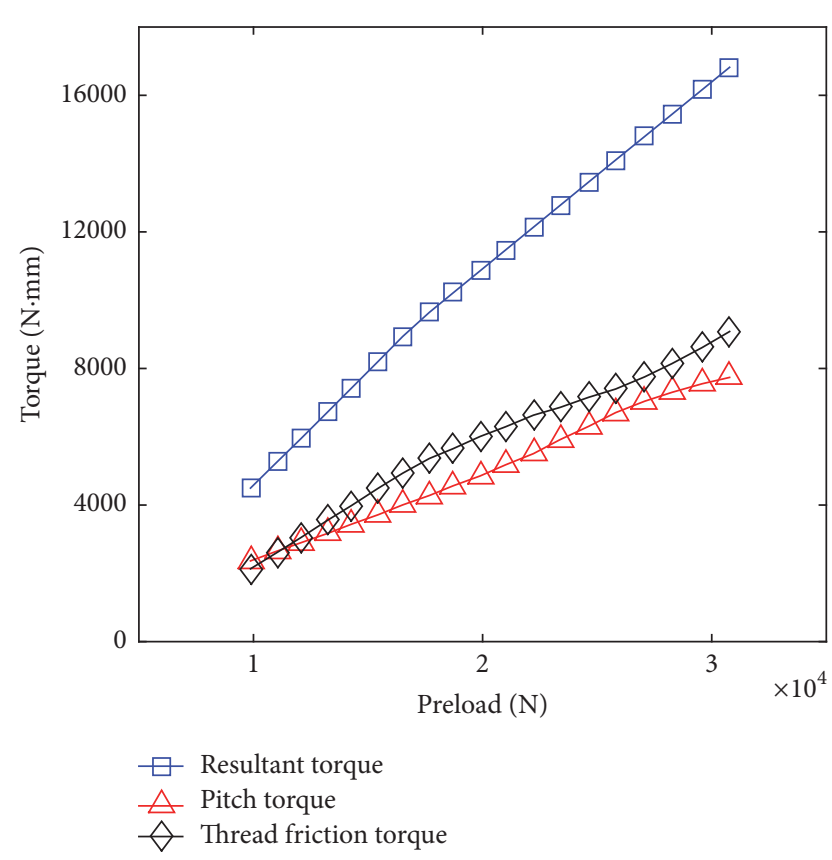

(a) Simulating the tightening process

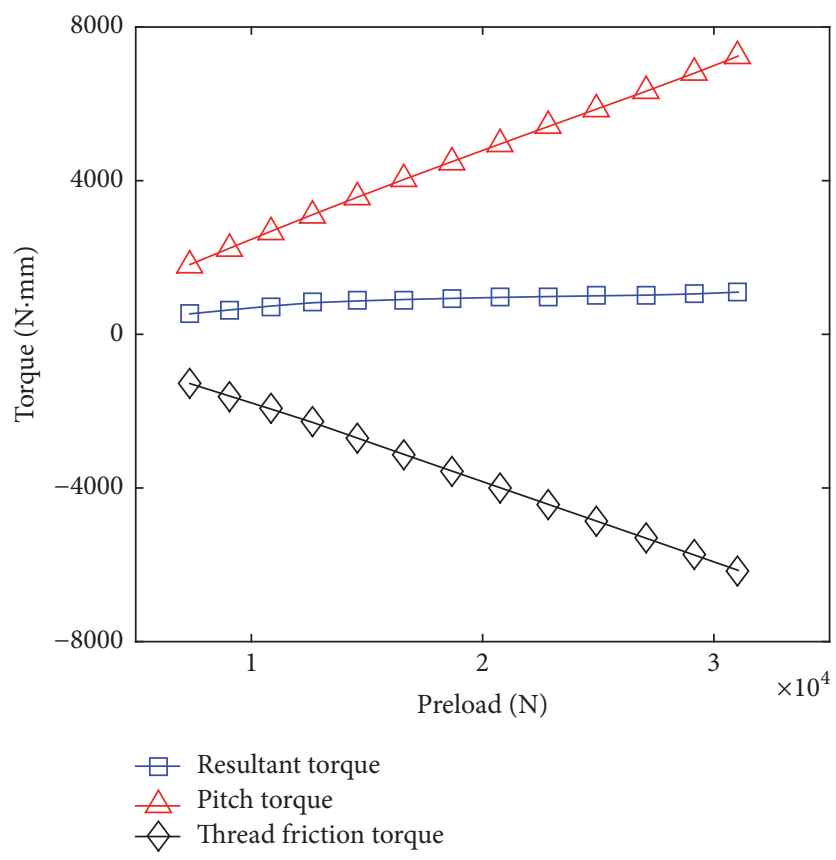

(b) Stretching the bolt

FIGURE 14: The relationships between different kinds of torque and preload.
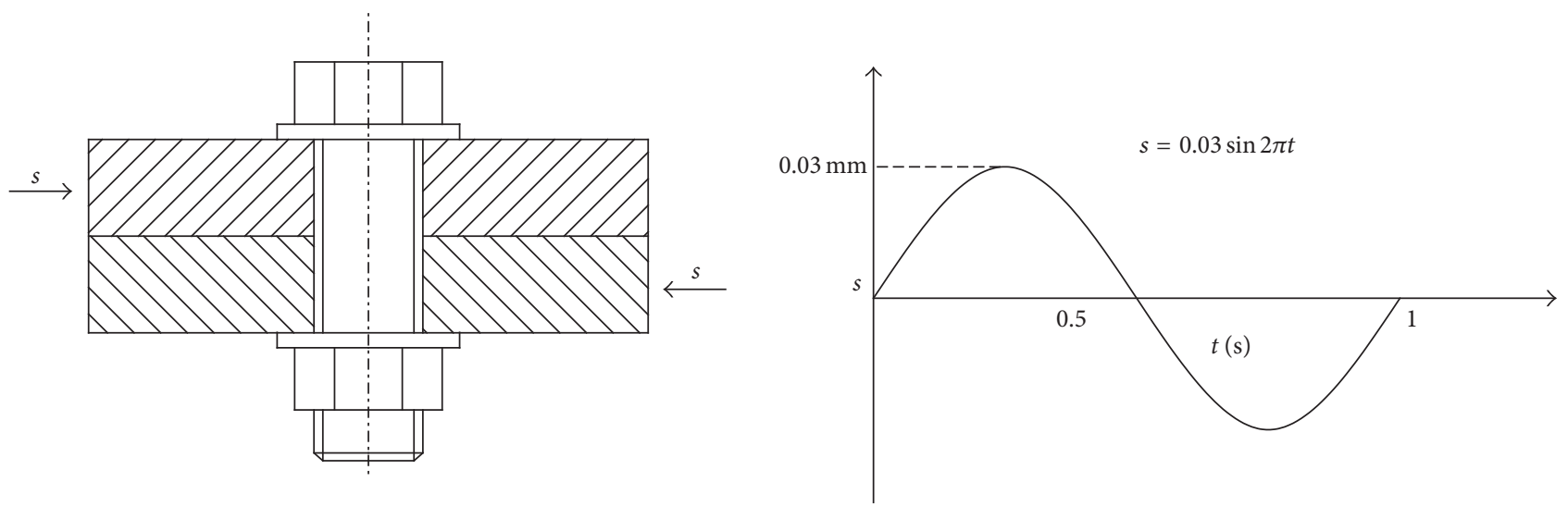

FIGURE 15: The diagrams of transversal harmonic load.

components. The evolutions of the preload of different fastening means during the first 15 load cycles are illustrated in Figure 16. It shows that, at the same load cycle, the selfloosening is much easier to appear and more preload gets lost when the preload is produced by the simplified way. This is because the resultant torque at contact surface is smaller.

It can be drawn from the above analysis that the approximate pretightening algorithm cannot take the place of the tightening process to study the self-loosening mechanism of bolt joints. To preform further analysis of the evolution of the preload during bolt self-loosening, the number of loading cycles is increased to 150 and the initial preload is set to $8 \mathrm{kN}$. The curve in Figure 17 displays the preload variation during the 150 cycles. It can be seen that, with the increase of the loading cycles, the variation curve of the preload tends to be flat after a rapid decline. The whole process can be roughly divided into two stages: the rapid decline stage and the flat stage, which is in accordance with the result of Junker.

4.2. Analysis of the Slip State at Contact Surfaces. The selfloosening behavior is mainly caused by slip at contact surfaces. Therefore, the dynamics during self-loosening is the main focus of the following analysis. This paper uses the relative motion of nodes to present the slip state, which is different from previous researches, and it is proved to be in greater detail by contrast with the traditional method using the friction relation.

The process of the preload variation mainly consists of two stages (exampled in Section 4.1). Taking the bolt head bearing surface as an example, the relative motion of nodes 


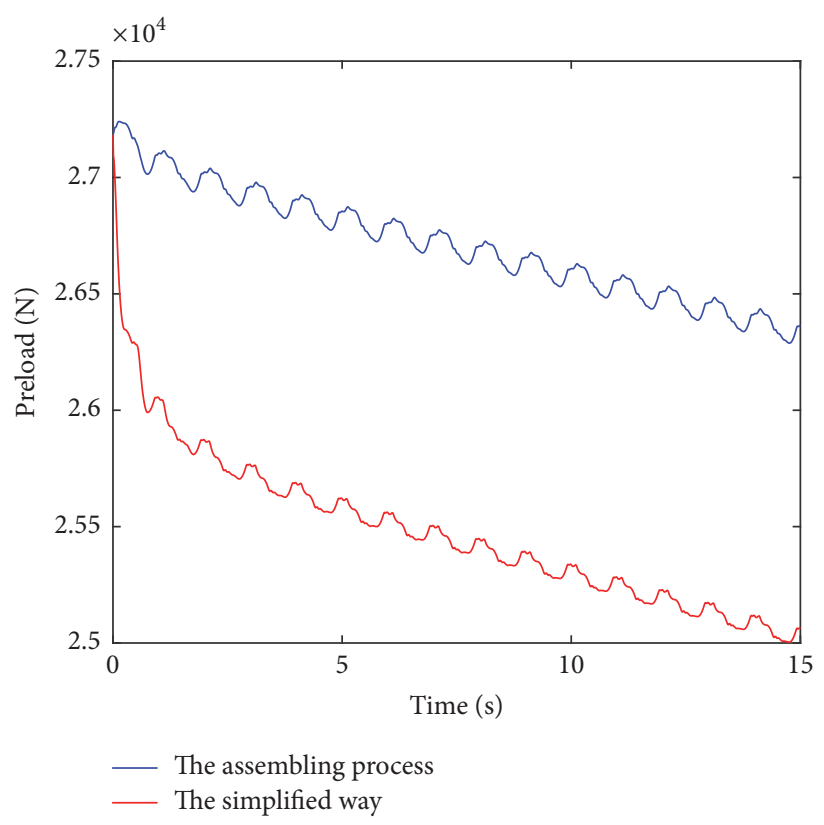

FIGURE 16: The preload variations under different fastening ways.

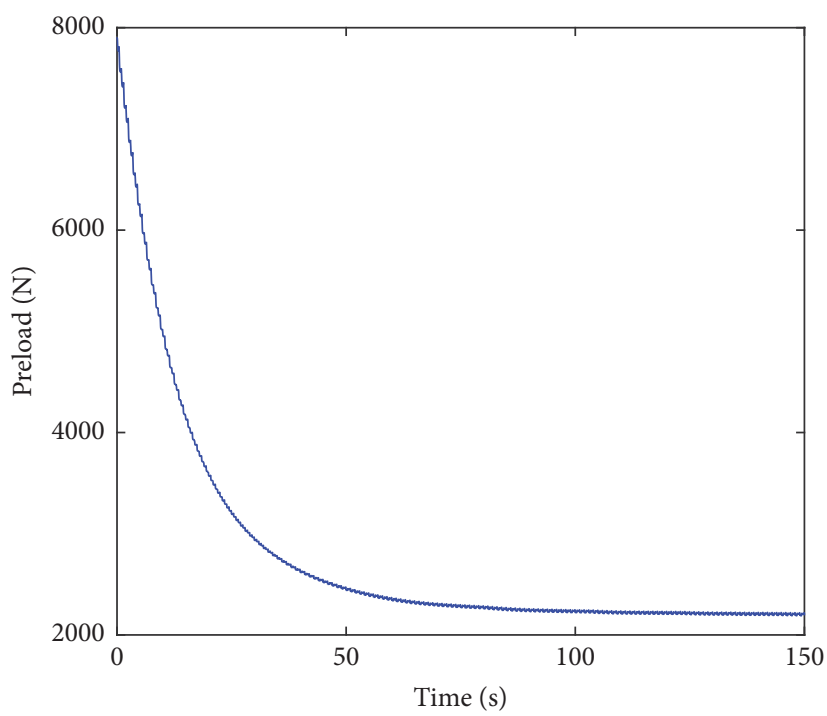

FigURE 17: The curve of preload variation during the 150 cycles.

during the two stages is analyzed. Because the clamped components are assumed to be rigid bodies, the relative rotation between contact surfaces is equivalent to the rotation of bolt around its axis. In the calculations, the uniform distribution nodes $1 \sim 8$ are selected from the outside edge of bolt and node $\mathrm{A}$ is the intersection point between the contact surface and the axis of bolt. The relative rotation at contact surface can be simplified to the rotation angle of nodes $1 \sim 8$ around node A (Figure 18).

According to Figure 19, the range of analysis time is $0 \sim 1 \mathrm{~s}$ (the decline stage) and 145 150 s (the flat stage). During the two stages, the rotation angle of each calculation node around node $\mathrm{A}$ is shown.
As shown before, all the nodes rotate along the loose direction as a whole which causes the preload loss. However, at the beginning of the self-loosening process, not all of the nodes rotate at the same time but one node rotates firstly and drives the rotation of the other nodes. Moreover, in the process of rotating, the rotation angles of some nodes are large and some are small. When the movement direction of clamped component changes, the rotation angles of those whose rotation angles are large previously begin to decrease. Meanwhile, the rotation angles of those whose rotation angles are small increase. This presents a creep slip phenomenon at contact surface under reversed cyclic load. With increase of the loading cycles, the preload continues to decline. And, 

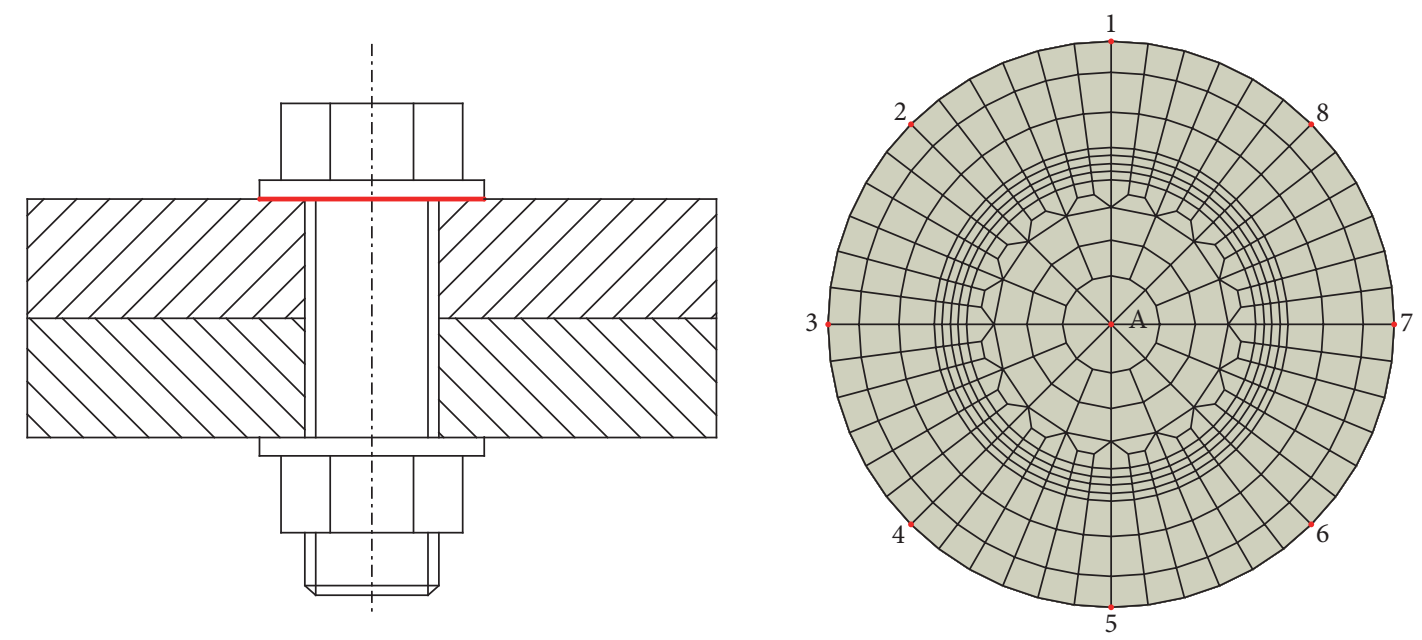

FIGURE 18: The diagrams of the contact surface and the calculation points.

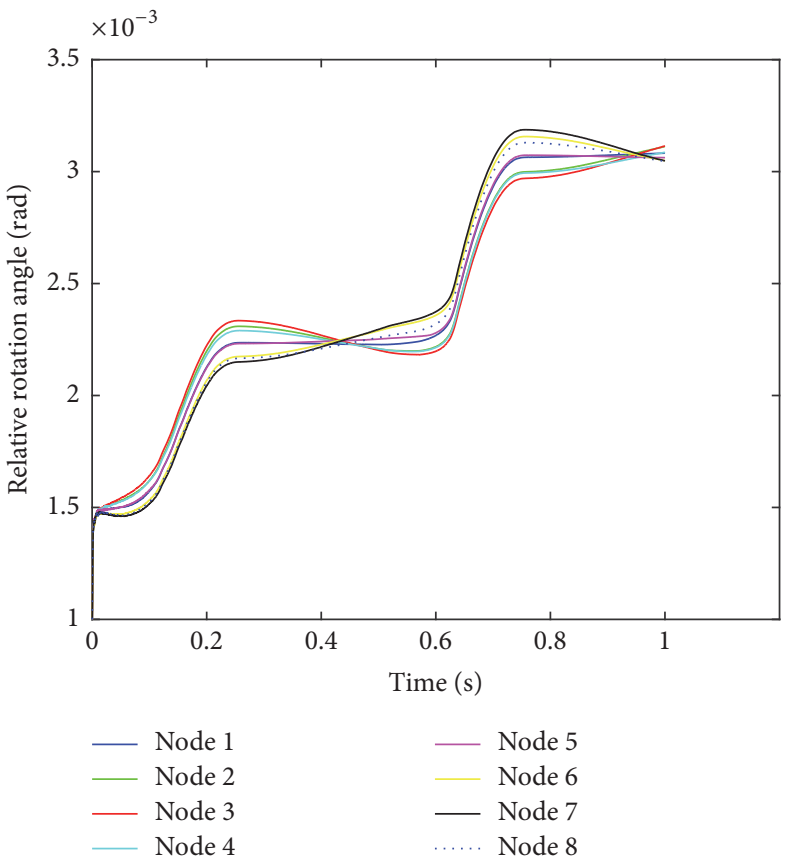

(a) The rapid decline stage

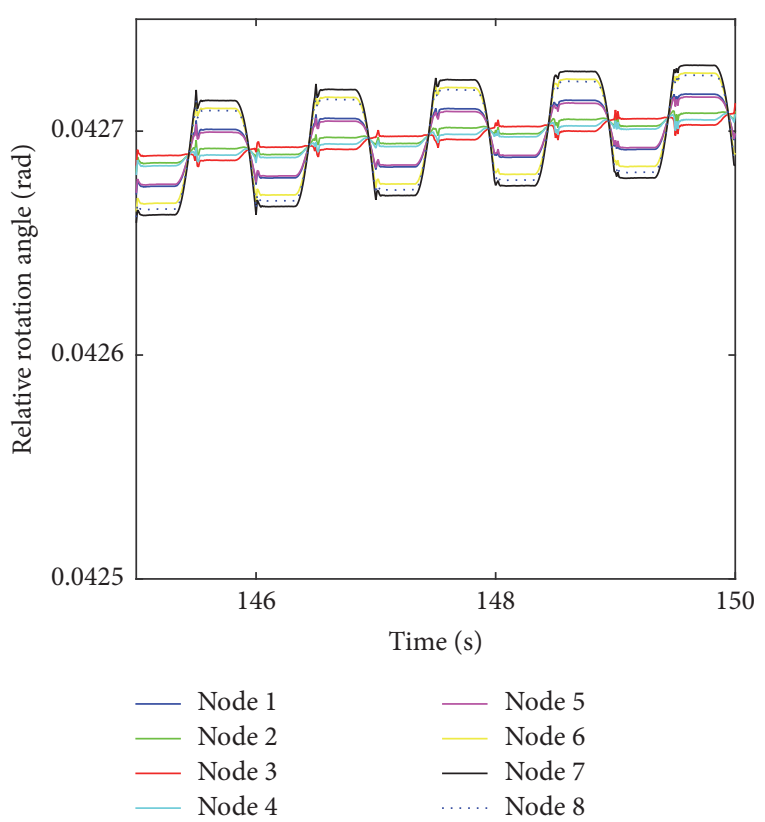

(b) The flat stage

FIGURE 19: The rotation angles of each point in different stages.

finally, all nodes present a back and forth rotation at one place, which causes the flat stage.

To analyze the slip state during the initial stage of selfloosening (when preload is $27.2 \mathrm{kN}$ ), all nodes along the outer edge are taken into account, and their relative rotation velocities around node $\mathrm{A}$ are carried out, as shown in Figure 20. Figure 21 shows the relative rotation velocity of each calculation node at some moments. It can be noted that the contact surface is slipping in a creep form. For comparison, the friction method is also applied in the analysis of the contact state. Based on the local key parameter $\eta_{n}$ calculated, the slip state contours are displayed in Figure 22. However, there is no significant difference among the three figures, which reflects that this method cannot give a detailed description of the slip state for a short time. Through the analysis of a whole cycle, it suggests that there are always two regions whose velocity directions are opposite. Owing to the continuity of motion, it means that there is a stick region on the contact surface at any moment, and bolt self-loosing can occur without complete slip on the bolt head bearing surface.

In addition, the relative motion between thread interfaces is analyzed in a similar way. Two helical segments are intercepted from the contact location of bolt and nut (Figure 23), respectively. The rotation velocities of nodes belonging to the two helical segments can be calculated to build the positionvelocity fields of bolt and nut at any time, and the velocity 


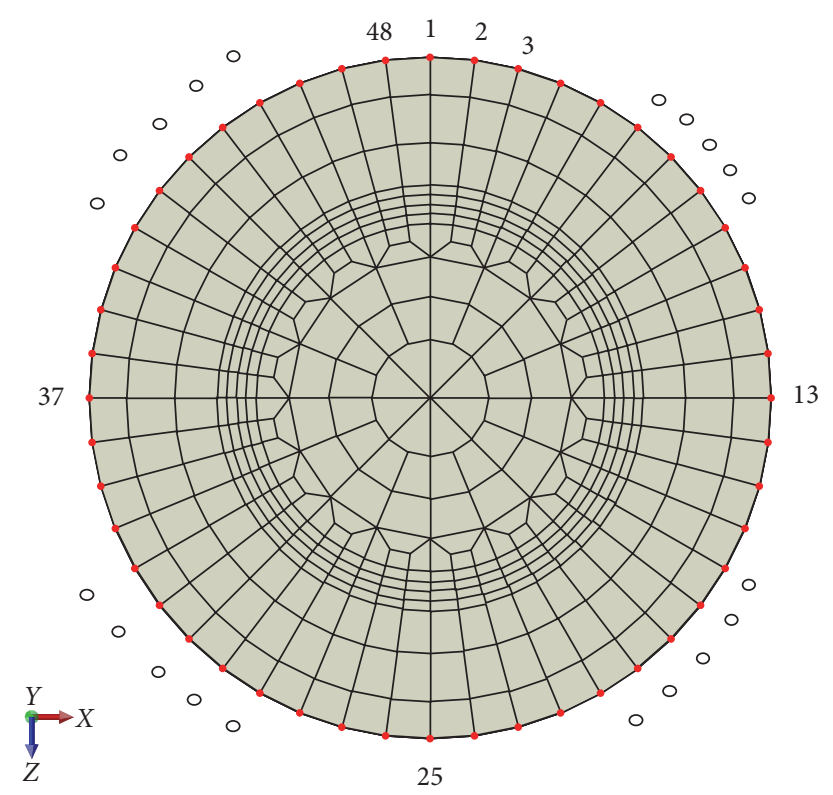

FIGURE 20: The node number along the outer edge.

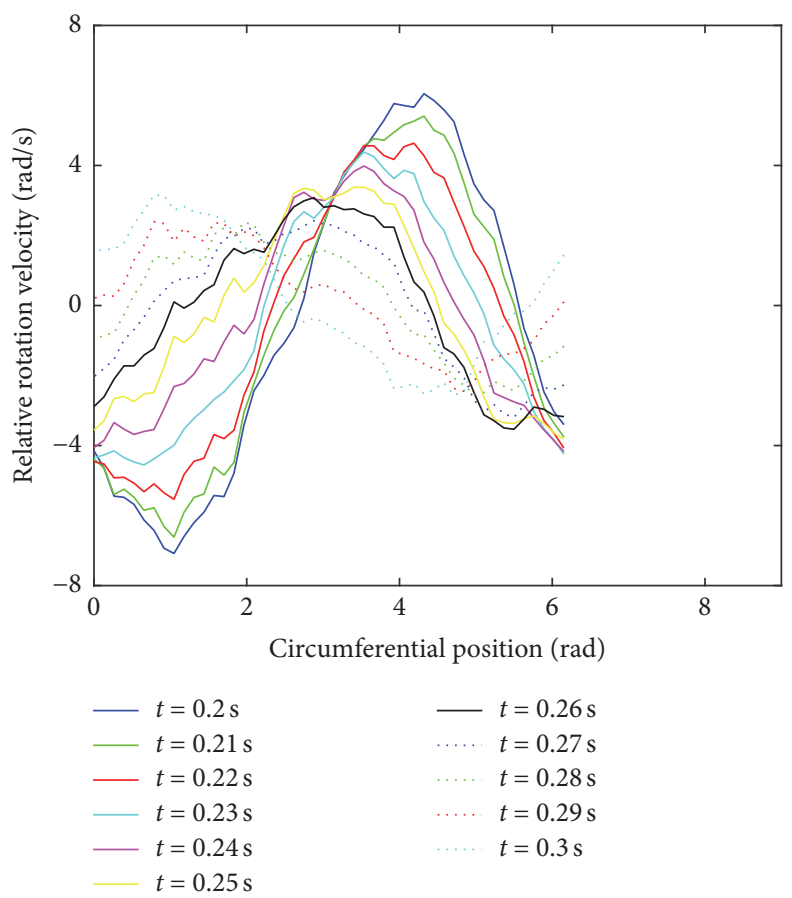

FIGURE 21: The relative rotation velocity of each node at different moment.

between adjacent nodes can be obtained approximately by linear interpolation. Figure 24 shows the position-velocity curve at $0.25 \mathrm{~s}$.

Based on the position-velocity curves of bolt and nut, the relative position-velocity relationship between thread interfaces can be acquired by subtracting them. Figure 25 shows the relative position-velocity relationships at some moments. It can be seen that all curves intersect the horizontal line that the value is zero. This means that there is always a stick region in the thread interfaces, which is consistent with the conclusion presented on the bolt head bearing surface. The slip state contours between thread interfaces are also given for comparison (Figure 26). However, there is still no significant difference among them.

To further strengthen the trust in the results summed before, the relation between transverse force (shear force) and transverse displacement during the initial fifteen cycles is shown in Figure 27. It is noted that the hysteresis loop 
$\eta_{n}$

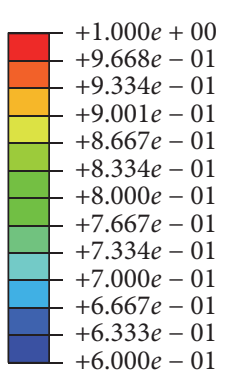

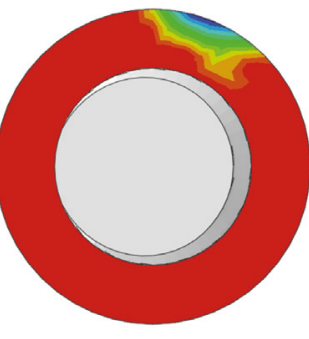

$t=0.2 \mathrm{~s}$

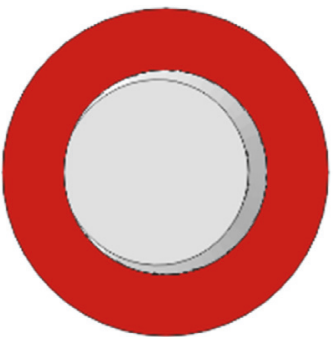

$t=0.25 \mathrm{~s}$

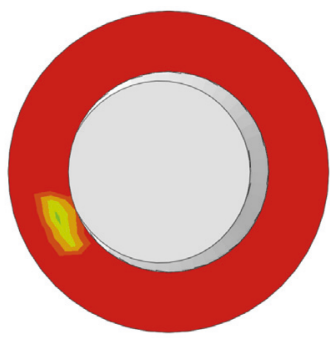

$t=0.3 \mathrm{~s}$

FIgURE 22: The slip state contours at different time.
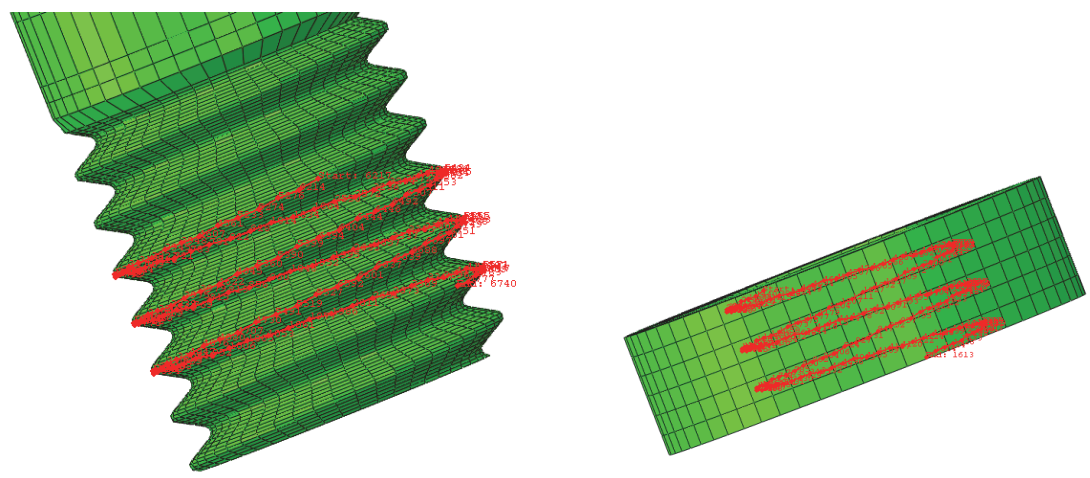

FIGURE 23: The schematic diagrams of helical segments.

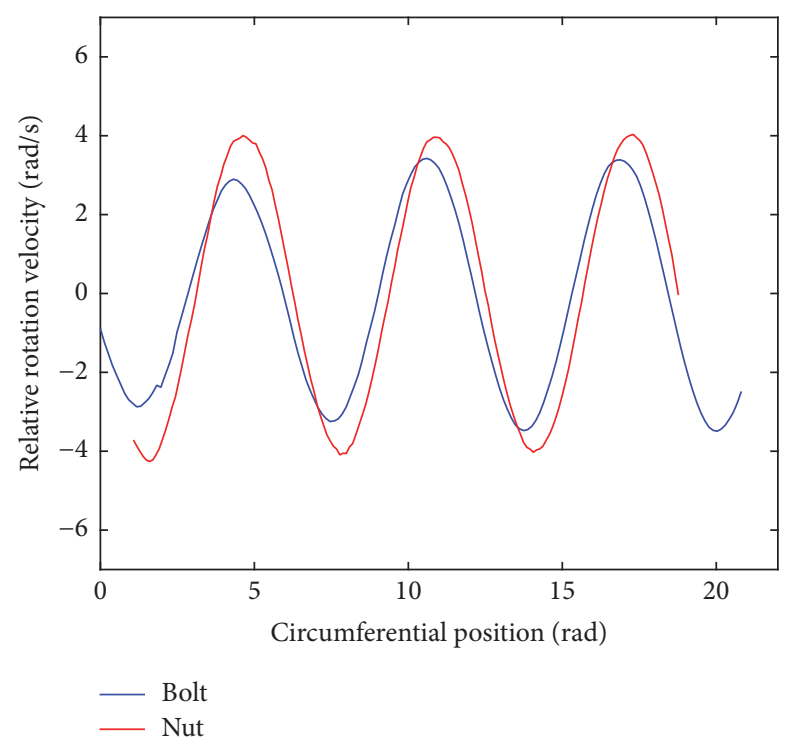

FIgURE 24: The position-velocity fields of bolt and nut at $0.25 \mathrm{~s}$.

only involves slope regions and has no flat region. The slope provides an indication of the joint stiffness in the transverse direction and the reduction in slope is a sign of slip at contact surfaces. However, in the slope region, the figure indicates that the contact surfaces undergo localized slip. No flat region means that the complete slip does not occur at contact surfaces during the initial self-loosening. This is consistent with the conclusion obtained by analyzing the relative motion of nodes.

\section{Conclusions}

The self-loosening process of bolt joints is investigated combining the tightening process by a three-dimensional 


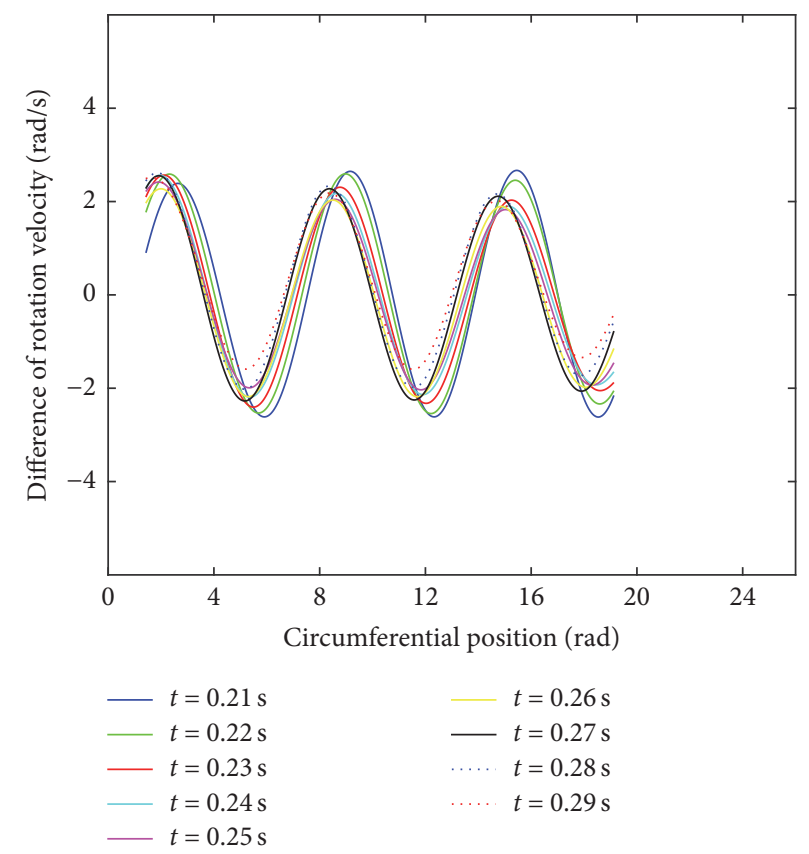

FIGURE 25: The relative rotation velocity of each node at different moment.

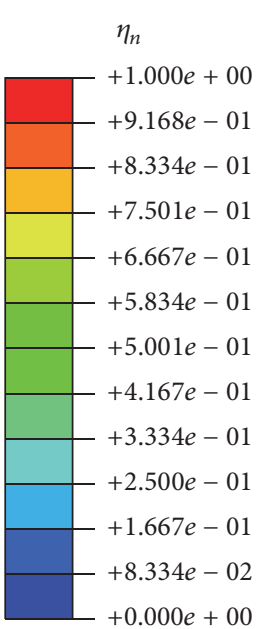

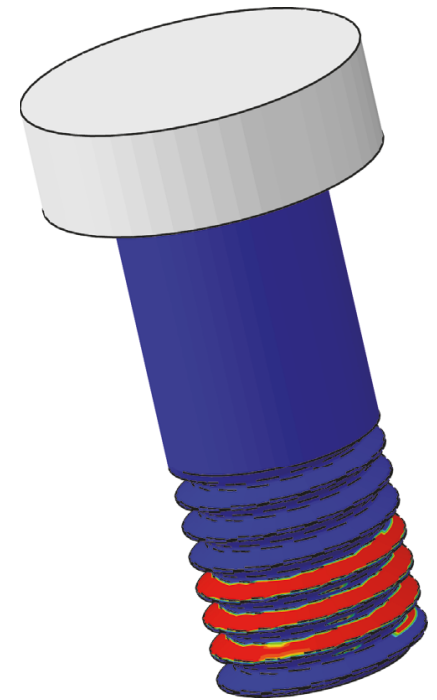

$t=0.2 \mathrm{~s}$

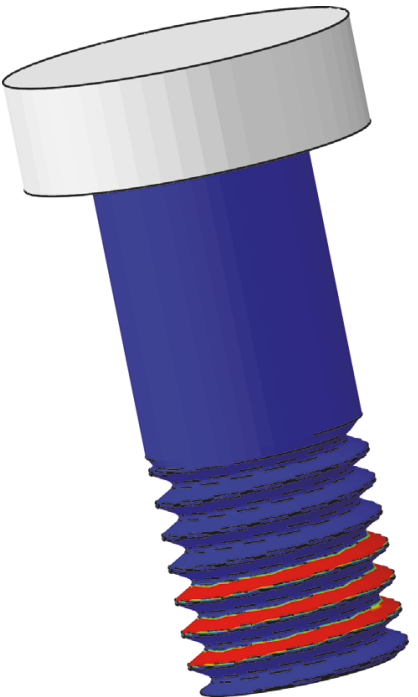

$t=0.25 \mathrm{~s}$

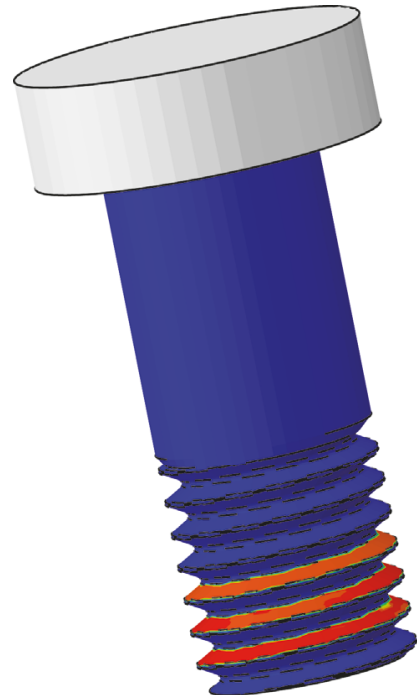

$t=0.3 \mathrm{~s}$

FIgURE 26: The slip state contours between thread interfaces.

finite element model in this paper. The FE model is meshed with hexahedral elements, and its accuracy is verified and validated compared with the analytical and experimental results. Followed by simulating different fastening means, the differences between them and their effects on bolt selfloosening are discussed. Finally, we utilize the relative motion of nodes to describe the contact states, and the conventional Coulomb friction method is also applied for contrast. Based on the FEA results, the following conclusions are drawn:

(1) Based on the mathematical expression, the threads are meshed with hexahedral elements by modifying the node coordinates of the cylindrical hexahedral meshes, which is proved to be effective. And a selfdeveloped plug-in is made for parametric modeling, and its functions can be expanded in further study.

(2) Through comparing with a simplified pretightening algorithm, it is demonstrated that the tightening process cannot be replaced, because the simplified way may cause a smaller resultant torque due to the opposite direction of the two torque components on the thread interface. For the same reason, it will lead 


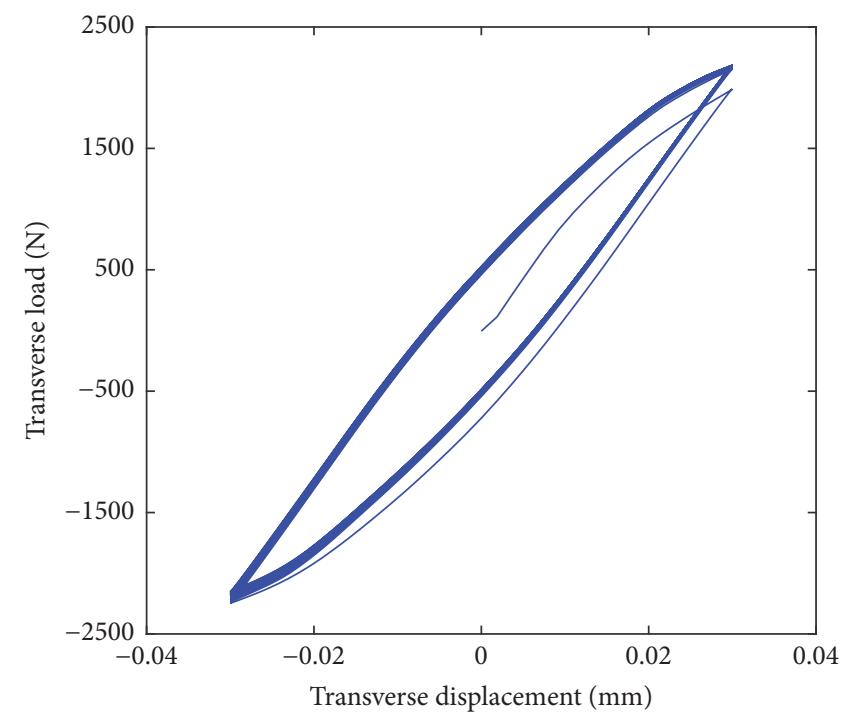

FIGURE 27: Hysteresis loops of transverse displacement and load.

to a greater loss of preload than the value in reality under the same number of load cycles.

(3) By contrast, the relative motion between nodes is found in a greater detail to describe the slip state at contact surfaces than Coulomb's law of friction. According to the simulation results of bolt selfloosening, it reveals that there exists a creep slip phenomenon on the bolt head bearing surface, which causes the bolt self-loosening to occur even when some contact facets are stuck.

\section{Conflicts of Interest}

The authors declare that there are no conflicts of interest regarding the publication of this paper.

\section{Acknowledgments}

The paper is supported by National Science and Technology Major Project of the Ministry of Science and Technology of China (no. 2011ZX02403), National Natural Science Foundation of China (no. 11302035 and no. 11272074), and the Fundamental Research Funds for the Central Universities.

\section{References}

[1] D. Liang and S.-F. Yuan, "Decision fusion system for bolted joint monitoring," Shock and Vibration, vol. 2015, Article ID 592043, 11 pages, 2015.

[2] L. Zhu, J. Hong, G. Yang, and X. Jiang, "Experimental study on initial loss of tension in bolted joints," Journal of Mechanical Engineering Science, vol. 230, no. 10, pp. 35-54, 2015.

[3] G. H. Junker, "New criteria for self-loosening of fasteners under vibration," Aircraft Engineering \&amp; Aerospace, vol. 44, no. 10, pp. 14-16, 1969.
[4] N. G. Pai and D. P. Hess, "Experimental study of loosening of threaded fasteners due to dynamic shear loads," Journal of Sound and Vibration, vol. 253, no. 3, pp. 585-602, 2002.

[5] N. G. Pai and D. P. Hess, "Three-dimensional finite element analysis of threaded fastener loosening due to dynamic shear load," Engineering Failure Analysis, vol. 9, no. 4, pp. 383-402, 2002.

[6] X. Yang and S. Nassar, "Analytical and Experimental Investigation of Self-Loosening of Preloaded Cap Screw Fasteners," Journal of Vibration and Acoustics, vol. 133, no. 3, p. 031007, 2011.

[7] G. Dinger and C. Friedrich, "Avoiding self-loosening failure of bolted joints with numerical assessment of local contact state," Engineering Failure Analysis, vol. 18, no. 8, pp. 2188-2200, 2011.

[8] S. Kasei, "A study of self-loosening of bolted joints due to repetition of small amount of slippage at bearing surface," Journal of Advanced Mechanical Design, Systems, and Manufacturing, vol. 1, no. 3, pp. 358-367, 2007.

[9] S. Izumi, M. Kimura, and S. Sakai, "Small Loosening of Bolt-nut Fastener Due to Micro Bearing-Surface Slip: A Finite Element Method Study," Journal of Solid Mechanics and Materials Engineering, vol. 1, no. 11, pp. 1374-1384, 2007.

[10] T. Yokoyama, M. Olsson, S. Izumi, and S. Sakai, "Investigation into the self-loosening behavior of bolted joint subjected to rotational loading," Engineering Failure Analysis, vol. 23, pp. 3543, 2012.

[11] Y. Fujioka and T. Sakai, "Rotating loosening mechanism of a nut connecting a rotary disk under rotating-bending force," Journal of Mechanical Design, vol. 127, no. 6, pp. 1191-1197, 2005.

[12] X. Jiang, Y. Zhu, J. Hong, X. Chen, and Y. Zhang, "Investigation into the loosening mechanism of bolt in curvic coupling subjected to transverse loading," Engineering Failure Analysis, vol. 32, pp. 360-373, 2013.

[13] S. A. Nassar and B. A. Housari, "Effect of thread pitch and initial tension on the self-loosening of threaded fasteners," Journal of Pressure Vessel Technology, Transactions of the ASME, vol. 128, no. 4, pp. 590-598, 2006.

[14] S. A. Nassar and B. A. Housari, "Study of the effect of hole clearance and thread fit on the self-loosening of threaded 
fasteners," Journal of Mechanical Design, vol. 129, no. 6, pp. 586594, 2007.

[15] S. A. Nassar and P. H. Matin, "Clamp load loss due to fastener elongation beyond its elastic limit," Journal of Pressure Vessel Technology, Transactions of the ASME, vol. 128, no. 3, pp. 379387, 2006.

[16] A. M. Zaki, S. A. Nassar, and X. Yang, "Effect of conical angle and thread pitch on the self-loosening performance of preloaded countersunk-head bolts," Journal of Pressure Vessel Technology, vol. 134, no. 2, pp. 566-571, 2013.

[17] S. A. Nassar and B. A. Housari, "Effect of thread pitch and initial tension on the self-loosening of threaded fasteners," Journal of Pressure Vessel Technology, vol. 128, no. 4, pp. 129-138, 2010.

[18] J. Mackerle, "Finite element analysis of fastening and joining: A bibliography (1990-2002)," International Journal of Pressure Vessels and Piping, vol. 80, no. 4, pp. 253-271, 2003.

[19] M. Zhang, Y. Jiang, and C.-H. Lee, "Finite element modeling of self-loosening of bolted joints," Journal of Mechanical Design, vol. 129, no. 2, pp. 218-226, 2007.

[20] R. I. Zadoks and D. P. R. Kokatam, "Investigation of the axial stiffness of a bolt using a three-dimensional finite element model," Journal of Sound and Vibration, vol. 246, no. 2, pp. 349373, 2001.

[21] S. Izumi, T. Yokoyama, M. Kimura, and S. Sakai, "Looseningresistance evaluation of double-nut tightening method and spring washer by three-dimensional finite element analysis," Engineering Failure Analysis, vol. 16, no. 5, pp. 1510-1519, 2009.

[22] S. Izumi, T. Yokoyama, A. Iwasaki, and S. Sakai, "Threedimensional finite element analysis of tightening and loosening mechanism of threaded fastener," Engineering Failure Analysis, vol. 12, no. 4, pp. 604-615, 2005.

[23] T. Fukuoka, M. Nomura, and Y. Morimoto, "Proposition of helical thread modeling with accurate geometry and finite element analysis," Journal of Pressure Vessel Technology, vol. 130, no. 1, pp. 135-140, 2008.

[24] T. Fukuoka, "Analysis of the tightening process of bolted joint with a tensioner using spring elements," Journal of Pressure Vessel Technology, Transactions of the ASME, vol. 116, no. 4, pp. 443-448, 1994.

[25] The standard of People's Republic of China, "GB/T 16823.21997 General rules of tightening for threaded fasteners," 1997 (Chinese). 


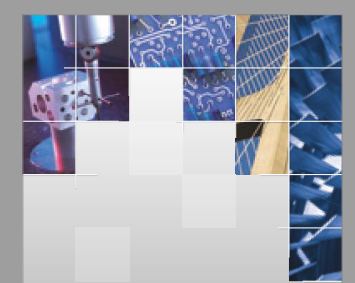

\section{Enfincering}
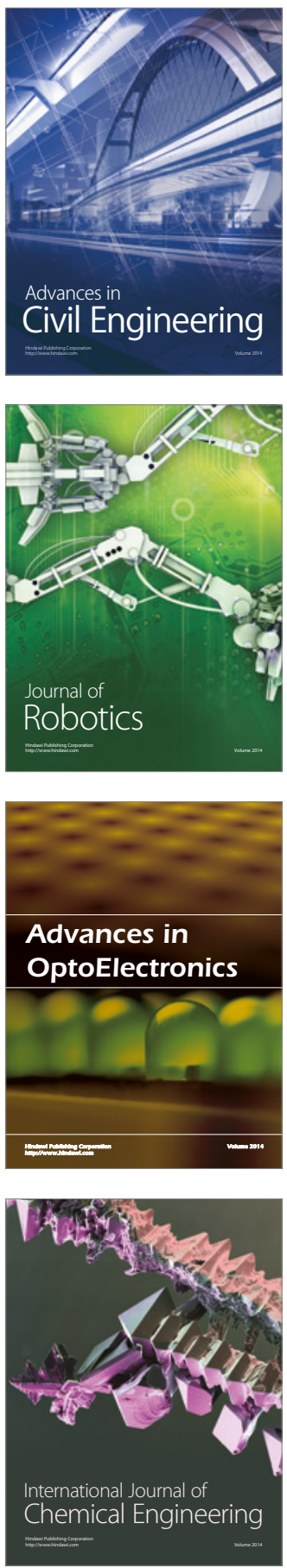

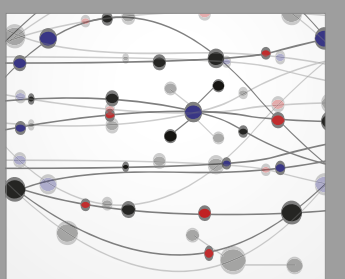

The Scientific World Journal

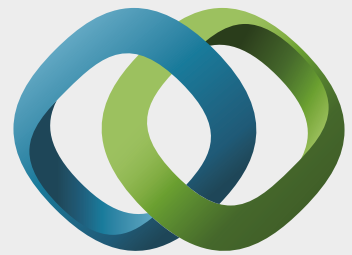

\section{Hindawi}

Submit your manuscripts at

https://www.hindawi.com
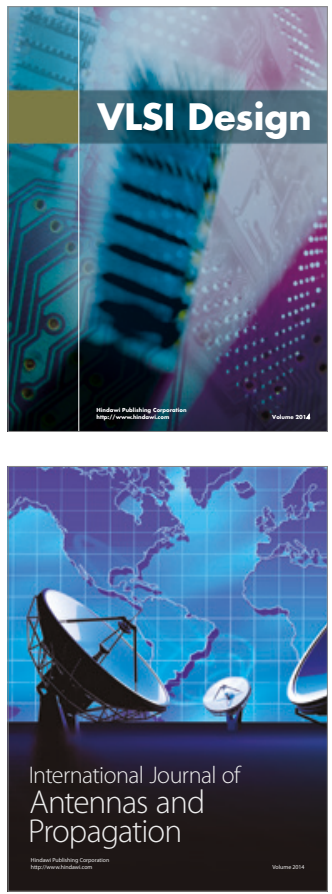

\section{Rotating}

Machinery
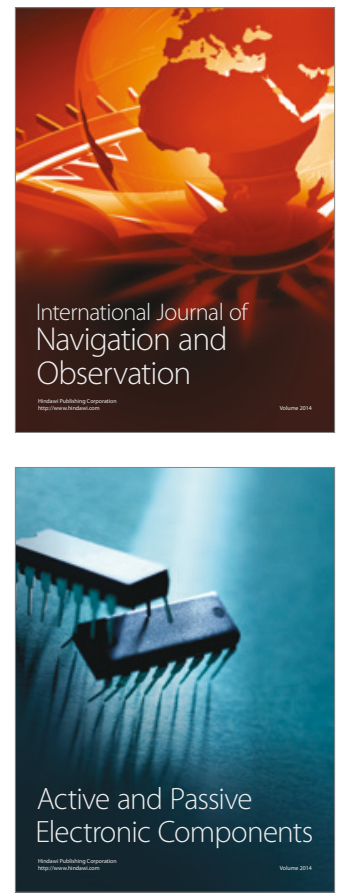
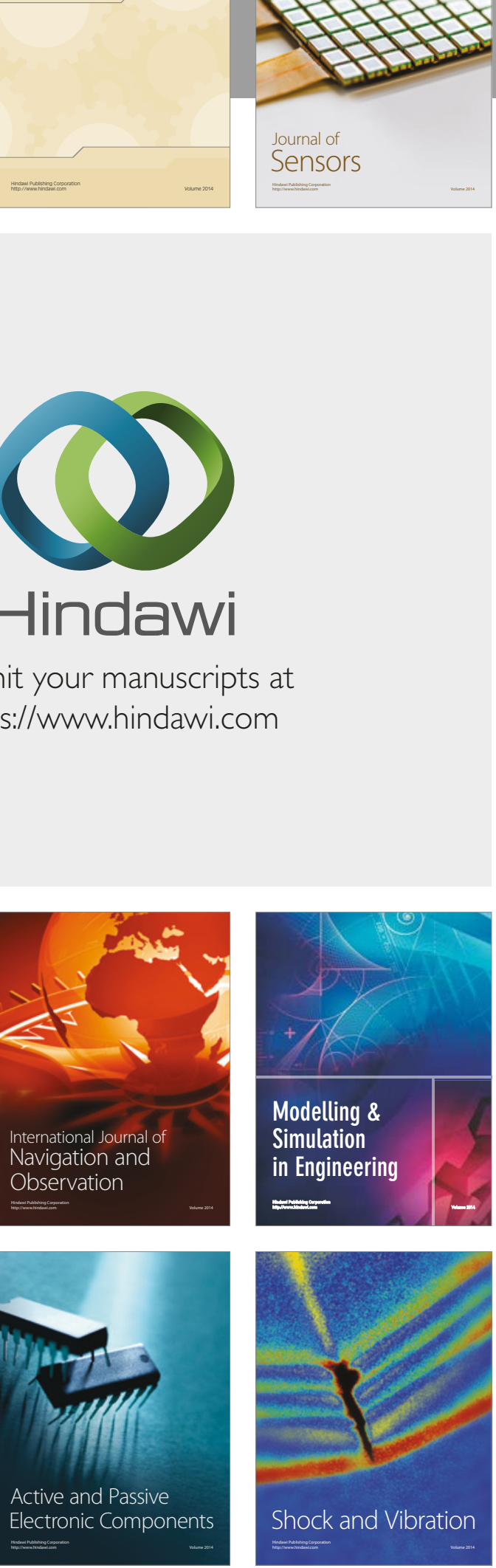
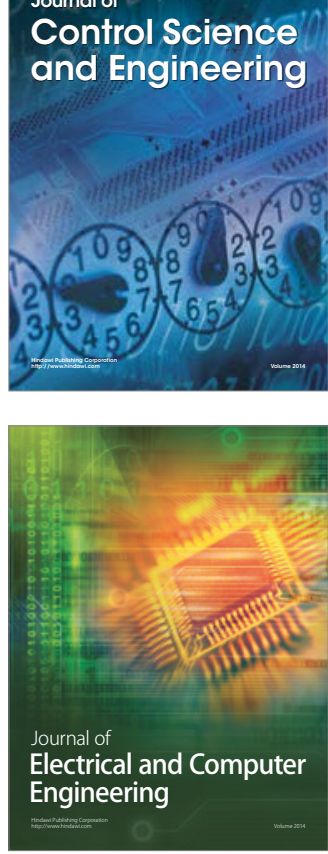

Distributed

Journal of

Control Science

and Engineering
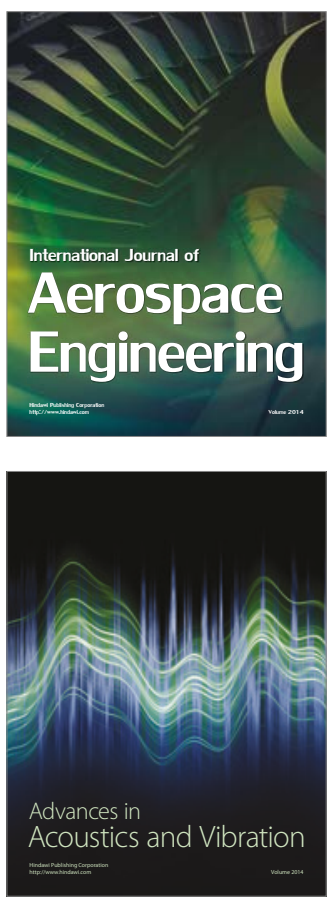

Sensor Networks 